\title{
Threefold Maximal Lepton Mixing and the Solar and Atmospheric Neutrino Deficits
}

PF Harrison, DH Perkins and WG Scott

December 1994 
DRAL is part of the Engineering and Physical Sciences Research Council

The Engineering and Physical Sciences Research Council does not accept any responsibility for loss or damage arising from the use of information contained in any of its reports or in any communication about its tests or investigations 
RAL-94-125

19 December 1994

\title{
Threefold Maximal Lepton Mixing and the Solar and Atmospheric Neutrino Deficits
}

\author{
P. F. Harrison \\ Physics Department, Queen Mary and Westfield College \\ Mile End Rd. London E1 4NS. UK ${ }^{1}$ \\ and \\ D. H. Perkins \\ Nuclear Physics Laboratory, University of Oxford \\ Keble Road, Oxford OX1 3RH. UK ${ }^{2}$ \\ and \\ W. G. Scott \\ Rutherford Appleton Laboratory \\ Chilton, Didcot, Oxon OX11 0QX. UK ${ }^{3}$
}

\begin{abstract}
Threefold maximal mixing would imply a cyclic permutation symmetry among the generations. The data from solar and atmospheric neutrino experiments are consistent with such mixing, and require a hierarchical spectrum of mass-squared differences for the neutrinos. A fit for the two independent masssquared differences (excluding only the HOMESTAKE solar neutrino result) yields: $\Delta m^{2}=(0.72 \pm 0.18) \times 10^{-2} \mathrm{eV}^{2}$ and $\Delta m^{\prime 2}<0.9 \times 10^{-11} \mathrm{eV}^{2}$ at $90 \%$ confidence. In the case that the neutrino mass spectrum is qualitatively similar to that of the charged leptons and quarks, these results can be re-expressed in terms of the neutrino masses as follows: $m_{3} \simeq 85 \pm 10 \mathrm{meV}$, and $m_{1}, m_{2}<3$ $\mu \mathrm{eV}$ at $90 \%$ confidence. Implications for future experiments are discussed.
\end{abstract}

Submitted to Physics Letters B

\footnotetext{
${ }^{1}$ E-mail:PFH@PH.QMW.AC.UK

${ }^{2}$ E-mail:PERKINS@PH.OX.AC.UK

${ }^{3} \mathrm{E}$-mail:SCOTTW@RL.AC.UK
} 
Data from solar [1] [2] [3] [4] and atmospheric [5] [6] [7] neutrino experiments strongly suggest that mixing occurs in the lepton sector, and furthermore that the corresponding mixing angles are large. In this paper we present the results of a phenomenological analysis of the combined available data on neutrino oscillations, including the data from accelerators and reactors, which aims to test the hypothesis that mixing in the lepton sector is (threefold) maximal. Such mixing would imply specific forms for the lepton mass matrices, corresponding to a cyclic permutation symmetry among the generations [8]. The mixing matrix can be written explicitly:

$$
U=\frac{1}{\sqrt{3}}\left(\begin{array}{lll}
\omega_{1} & \omega_{1} & \omega_{1} \\
\omega_{1} & \omega_{2} & \omega_{3} \\
\omega_{1} & \omega_{3} & \omega_{2}
\end{array}\right)
$$

where $U U^{\dagger}=1$ and the $\omega_{i}(i=1-3)$ are the complex cube-roots of unity. The mixing is maximal in that all the elements of the mixing matrix have equal modulus $\left(\left|U_{\nu l}\right|=\right.$ $1 / \sqrt{3}$ ). All unitarity triangles are equilateral (and congruent) and the convention independent CP violation parameter $J_{C P}[9]$ is extremal: $\left|J_{C P}\right|=1 /(6 \sqrt{3})$. In contrast to the case of the quarks, evolution with energy due to quantum corrections, is expected to have a negligible effect on the lepton mixing matrix [8]. A lepton mixing matrix of the above form has been considered previously by a number of authors [10].

Vacuum neutrino oscillations are conveniently discussed in a weak basis which diagonalises the mass-matrix of the charged leptons. At high energy, the matrix $A$ of normalised transition amplitudes $A_{l^{\prime} l}$ from a charged lepton state $l,(l=e, \mu, \tau)$ to a charged lepton state $l^{\prime}$ is given by: $A=\exp \left(-i m^{2} L / 2 E\right)$, where $m m^{\dagger} \equiv m^{2}$ is the hermitian-square of the neutrino mass matrix, $E$ is the neutrino energy and $L$ is the distance of propagation. In the case of maximal mixing the neutrino mass matrix in this basis can be written as a circulant [8], so that the resulting matrix of transition amplitudes (which depends on $L / E$ ) is then also circulant:

$$
A=\left(\begin{array}{lll}
p & q & r \\
r & p & q \\
q & r & p
\end{array}\right) .
$$

In terms of probabilities, $P\left(l \rightarrow l^{\prime}\right)=\left|A_{l^{\prime} l}\right|^{2}$ :

$$
\begin{aligned}
& P(e \rightarrow e)=P(\mu \rightarrow \mu)=P(\tau \rightarrow \tau)=P(\bar{e} \rightarrow \bar{e})=P(\bar{\mu} \rightarrow \bar{\mu})=P(\bar{\tau} \rightarrow \bar{\tau})=|p|^{2} \\
& P(e \rightarrow \mu)=P(\mu \rightarrow \tau)=P(\tau \rightarrow e)=P(\bar{e} \rightarrow \bar{\tau})=P(\bar{\tau} \rightarrow \bar{\mu})=P(\bar{\mu} \rightarrow \bar{e})=|q|^{2} \\
& P(e \rightarrow \tau)=P(\tau \rightarrow \mu)=P(\mu \rightarrow e)=P(\bar{e} \rightarrow \bar{\mu})=P(\bar{\mu} \rightarrow \bar{\tau})=P(\bar{\tau} \rightarrow \bar{e})=|r|^{2}
\end{aligned}
$$

where $P\left(l \rightarrow l^{\prime}\right)=P\left(\bar{l}^{\prime} \rightarrow \bar{l}\right)$, by CPT. Remarkably the various survival probabilities $P(l \rightarrow l)$, as measured in disappearance experiments, are identical, so that $P(l \rightarrow l)$ is a universal function of $L / E$, independent of generation. The appearance probabilities 
$P\left(l \rightarrow l^{\prime}\right)$ for the 'clockwise' transitions, eq. (4), are also all identical, as are those for the 'anticlockwise' transitions, eq. (5). The asymmetry between the clockwise and the anticlockwise transitions is $\mathrm{T}$ and $\mathrm{CP}$ violating and (if the neutrino masses are not degenerate) reaches $\pm 100 \%$, for particular values of $L / E$ (see eq. (7)-(8) below). The $L / E$ dependence of the complex amplitudes $p, q$ and $r$ is given by:

$$
\begin{aligned}
& |p|^{2}=\frac{1}{3}+\frac{2}{9}\left(c_{12}+c_{23}+c_{31}\right) \\
& |q|^{2}=\frac{1}{3}-\frac{1}{9}\left(c_{12}+c_{23}+c_{31}\right)+\frac{1}{3 \sqrt{3}}\left(s_{12}+s_{23}+s_{31}\right) \\
& |r|^{2}=\frac{1}{3}-\frac{1}{9}\left(c_{12}+c_{23}+c_{31}\right)-\frac{1}{3 \sqrt{3}}\left(s_{12}+s_{23}+s_{31}\right)
\end{aligned}
$$

where $c_{12}=\cos \left(\Delta m_{12}^{2} L / 2 E\right), s_{12}=\sin \left(\Delta m_{12}^{2} L / 2 E\right), \Delta m_{12}^{2}=m_{1}^{2}-m_{2}^{2}$ etc. and the $m_{i}$ are the neutrino masses: $m_{1} \leq m_{2} \leq m_{3}$. The three mass-squared differences are not independent: $\Delta m_{12}^{2}+\Delta m_{23}^{2}+\Delta m_{31}^{2}=0$. In eq. (7)-(8), the coefficient of the sine terms (which are CP violating) is $\pm 2 J_{C P}$. Clearly $|p|^{2}+|q|^{2}+|r|^{2}=1$, for all $L / E$, as required by conservation of probability.

Detailed predictions depend on the character of the neutrino mass spectrum. We consider first the case that the neutrino mass spectrum exhibits a pronounced hierarchy, similar to that of the charged leptons and the quarks, so that: $\Delta m_{23}^{2} \simeq-\Delta m_{31}^{2}$ ( $\left.\equiv \Delta m^{2}\right)$ and $\Delta m_{12}^{2}\left(\equiv \Delta m^{\prime 2}\right) \ll \Delta m^{2}$. At small values of $L / E$, ie. for $L / E \ll$ $\left(\Delta m^{2} / 2\right)^{-1}$, all the $L / E$-dependent phases are small so that $c_{12} \simeq c_{23} \simeq c_{31} \simeq 1$ and $s_{12} \simeq s_{23} \simeq s_{31} \simeq 0$. It follows that: $|p|^{2} \simeq 1$ and $|q|^{2} \simeq|r|^{2} \simeq 0$ initially, corresponding to no oscillation effects, with lepton number apparently conserved. If $L / E$ is increased so that: $\left(\Delta m^{2} / 2\right)^{-1} \ll L / E \ll\left(\Delta m^{\prime 2} / 2\right)^{-1}$, one phase remains small and two phases become appreciable, but equal and opposite: $s_{12} \simeq 0, s_{23} \simeq-s_{31}$ (ie. no significant $\mathrm{CP}$ violation) with $c_{12} \simeq 1, c_{23} \simeq c_{31}$, so that:

$$
\begin{aligned}
& |p|^{2} \simeq \frac{5}{9}+\frac{4}{9} \cos \left(\Delta m^{2} L / 2 E\right) \\
& |q|^{2} \simeq|r|^{2} \simeq \frac{2}{9}-\frac{2}{9} \cos \left(\Delta m^{2} L / 2 E\right) .
\end{aligned}
$$

In the limit (see below) that the cosine terms average to zero: $|p|^{2}=5 / 9$ and $|q|^{2}=$ $|r|^{2}=2 / 9$. Finally, if $L / E \gg\left(\Delta m^{\prime 2} / 2\right)^{-1}$, all three phases become appreciable and in the limit: $|p|^{2}=|q|^{2}=|r|^{2}=1 / 3$.

The approach to the above limits will depend on the degree of $L / E$ averaging in the experiment. For any fixed fractional $L / E$ range, the oscillations will always average to zero for sufficiently large $L / E$. In many of the cases of interest here, the $L / E$ range sampled is relatively wide. The effect of the averaging can then be approximated by taking the fractional $L / E$ range to be $\pm 100 \%$ (ie. all $L / E$ from zero to twice the mean 
contribute equally), so that, for example, eq. (9)-(10) become:

$$
\begin{aligned}
& |p|^{2}=\frac{5}{9}+\frac{4}{9} \frac{\sin \left(\Delta m^{2} L / E\right)}{\Delta m^{2} L / E} \\
& |q|^{2}=|r|^{2}=\frac{2}{9}-\frac{2}{9} \frac{\sin \left(\Delta m^{2} L / E\right)}{\Delta m^{2} L / E} .
\end{aligned}
$$

For $L / E \ll\left(\Delta m^{2} / 2\right)^{-1}$, eq. (11)-(12) give $|p|^{2}=1$ and $|q|^{2}=|r|^{2}=0$, while for $L / E \gg\left(\Delta m^{2} / 2\right)^{-1},|p|^{2}=5 / 9$ and $|q|^{2}=|r|^{2}=2 / 9$, as expected. Residual oscillations are very small, and the two plateau regions are separated by a well localised threshold at $L / E \simeq\left(\Delta m^{2}\right)^{-1}$. A similar threshold at $L / E \simeq\left(\Delta m^{\prime 2}\right)^{-1}$, will mark the descent to the third plateau region characterised by $|p|^{2}=|q|^{2}=|r|^{2}=1 / 3$. Note that the CP violating terms, which are anyway significant only beyond the second threshold, ie. for $L / E \gtrsim\left(\Delta m^{2}\right)^{-1}$, tend to zero in the limit of complete averaging.

The most readily testable predictions are those related to the universality of the survival probability $P(l \rightarrow l)$ and its $L / E$ dependence. Results from disappearance experiments are summarised in Table 1.

Accelerator experiments have explored the region $L / E \lesssim 1 \mathrm{~km} / \mathrm{GeV}$. The CDHSSPS [11] results come from neutrino-nucleon cross-section measurements in a highenergy $\nu_{\mu}$-beam; to obtain probabilities we have divided by theoretical cross-sections [12], based on nucleon structure-functions measured using charged-lepton beams. The CHARM-PS [13] result, which also refers to $\nu_{\mu}$, was normalised internally by comparing muon event rates in two similar detectors at different distances. The KARMEN [14] experiment measures the cross section for the reaction $C^{12}\left(\nu_{e}, e\right) N^{12}$ using $\nu_{e}$ from muon decay; we give the ratio of measured to predicted cross-sections (the average of three predictions [14] is taken, with a spread of $\pm 6 \%$ ). The accelerator results show no evidence for oscillation effects, and assuming maximal mixing, imply $\Delta m^{2} \lesssim 1 \mathrm{eV}^{2}$.

Reactor experiments detect $\bar{\nu}_{e}$ produced by $\beta$-decay of neutron-rich fission fragments and explore the $L / E$ range $2 \lesssim L / E \lesssim 50 \mathrm{~m} / \mathrm{MeV}$. The ILL/GOSGEN [15], BUGEY [16] and KRASNOYARSK [17] results given in Table 1 are averaged over the reactor neutrino spectrum. The ILL/GOSGEN and BUGEY experiments measure the energy $E_{e}$ of the positron produced by the inverse $\beta$-decay of a free proton ( $E=E_{e}+1.8 \mathrm{MeV}$ ), so that these results can be binned more finely as a function of neutrino energy, if required (see below). The reactor results are also consistent with no oscillations, and assuming maximal mixing, imply $\Delta m^{2} \lesssim 10^{-2} \mathrm{eV}^{2}$.

The atmospheric neutrino data relate to neutrinos produced by cosmic ray interactions in the atmosphere, and cover an extremely wide range in $L / E: 2 \lesssim L / E \lesssim 2 \times 10^{4}$ $\mathrm{km} / \mathrm{GeV}$. The best-determined quantity for atmospheric neutrinos is the double ratio: $R \equiv(\mu / e)_{D A T A} /(\mu / e)_{M C}$, where $(\mu / e)_{D A T A}$ is the observed ratio of muon to electron events in the detector, and $(\mu / e)_{M C}$ is the expected muon to electron ratio, assuming 
no oscillations, and accounting for experimental cuts and efficiencies. The flux ratio $\phi\left(\nu_{\mu}+\bar{\nu}_{\mu}\right) / \phi\left(\nu_{e}+\bar{\nu}_{e}\right)\left(\equiv \nu_{\mu} / \nu_{e}\right)$ needed to calculate the double ratio is known to an accuracy of $\pm 5 \%$ and is close to $2 / 1$ (at least for $E<2 \mathrm{GeV}$ ), as one might naively expect. The KAMIOKA [5] group, using a water Cerenkov detector, were the first to report an anomaly in the behaviour of atmospheric neutrinos, obtaining a value for $R$ significantly less than unity, for fully contained events. Although absolute fluxes are difficult to calculate reliably, there are strong indications [18] that the rate of $\nu_{e}$ events agrees with expectations, and that it is the disappearance of $\nu_{\mu}$ which leads to the anomaly in the double ratio. In Table 1 , therefore, we quote the double ratio $R$ directly, for the atmospheric neutrino experiments, as a first estimate for the survival probability for $\nu_{\mu}$, although we anticipate that there will be corrections to this.

The most recent KAMIOKA data [5] comprise two independent data-sets: the sub$\mathrm{GeV}$ data-set $(E \simeq 0.5 \mathrm{GeV}$ ), restricted to fully contained events and the multi-GeV data-set $(E \simeq 5 \mathrm{GeV}$ ), which includes partially contained events. The KAMIOKA group have plotted the data as a function of $\cos \theta$, where $\theta$ is the zenith angle of the outgoing lepton. At high energy we expect the outgoing lepton direction to be well correlated with the incoming neutrino direction. For the multi-GeV data-set, therefore, we estimate the propagation distance $L$ for each data point as a function of $\cos \theta$ using $L=\sqrt{R_{\oplus}^{2} \cos ^{2} \theta+2 R_{\oplus} H+H^{2}}-R_{\oplus} \cos \theta$, where $R_{\oplus}=6400 \mathrm{~km}$ is the radius of the Earth and $H \simeq 20 \mathrm{~km}$ is the effective height of the atmosphere. At low energies, on the other hand, we expect the correlation between the lepton direction and the neutrino direction to be very weak, and for the sub-GeV data set we have combined the KAMIOKA data points to yield two averaged data points corresponding to the upward $(\cos \theta<0)$ and downward $(\cos \theta>0)$ hemispheres respectively (see Table 1$)$. Confirmation of the atmospheric neutrino anomaly comes from the IMB [7] water Cerenkov detector and from the SOUDAN experiment [6], using a tracking detector. Two other tracking experiments, FREJUS [19] and NUSEX [20], have reported no effect. The IMB, FREJUS, NUSEX and SOUDAN results quoted in Table 1, refer to fully contained events, averaged over all zenith angles. Finally, we note that related data on upward going muons have also been cited as evidence for (and against) neutrino oscillations; in view of large uncertainties in the the expected rates [21] however, we have not considered these data here.

The solar neutrino data cover the range $10^{10} \lesssim L / E \lesssim 10^{12} \mathrm{~m} / \mathrm{MeV}$ and relate to $\nu_{e}$ produced in fusion processes in the solar core. The results quoted in Table 1 are in each case the measured rate [22] divided by the expected rate, calculated as the mean of the Bahcall-Pinsonneault and Turck-Chieze predictions of the Standard Solar Model [23]. The KAMIOKA [4] experiment detects neutrino-electron scattering and is sensitive to the highest energy (boron-8) neutrinos with $E \gtrsim 7.5 \mathrm{MeV}$. The 
HOMESTAKE experiment [1] using a chlorine target, is also predominantly sensitive to boron-8 neutrinos, but with a lower energy threshold ( $E \gtrsim 0.81 \mathrm{MeV}$ ). Most of the signal from the two gallium experiments SAGE [3] and GALLEX [2] ( $E \gtrsim 0.23 \mathrm{MeV})$ comes from p-p neutrinos.

The data from all the above experiments are plotted in Figure 1, as a function of $L / E$. A number of interesting features are immediately apparent. The data taken together strongly suggest the existence of an abrupt threshold in the region $L / E \simeq$ $20-80 \mathrm{~km} / \mathrm{GeV}$ (corresponding to $\Delta m^{2} \sim 10^{-2} \mathrm{eV}^{2}$ ), marking the onset of a distinct constant plateau. The plateau comprises all the atmospheric data with $L / E>80$ $\mathrm{km} / \mathrm{GeV}$ (with the possible exception of the last KAMIOKA multi-GeV point) and all the solar data (with the exception of the HOMESTAKE point). Furthermore the plateau height suggested by the data seems to be close to the prediction $P(l \rightarrow l)=$ $5 / 9 \simeq 0.56$, characteristic of maximal mixing. The solid curve, which adequately describes most of the data, is the maximal mixing prediction eq. (11), with $\Delta m^{2}=$ $0.72 \times 10^{-2} \mathrm{eV}^{2}$ (see below for details of fits).

For the atmospheric neutrino data a more detailed analysis is necessary to account for the fact that the initial fluxes comprise both $\nu_{\mu}$ and $\nu_{e}$. If mixing occurs, the loss of $\nu_{\mu}$ is then partly compensated by the effect of $\nu_{e} \rightarrow \nu_{\mu}$ oscillations and vice-versa. In the maximal mixing scenario, from eq. (9)-(10), we expect $P(e \rightarrow \mu)=(1-P(\mu \rightarrow$ $\mu)) / 2$ and $P(\mu \rightarrow e)=(1-P(e \rightarrow e)) / 2$. In the approximation that the initial flux ratio $\nu_{\mu} / \nu_{e}=2 / 1$ (valid for all the contained event data, $E \lesssim 2 \mathrm{GeV}$ ), the rate of $\nu_{e}$ will be unaffected by the oscillations, while the reduction factor applying to $\nu_{\mu}$ becomes $2 / 3$ instead of $5 / 9$. More generally, the survival probability $P(\mu \rightarrow \mu)$ is related to the measured double ratio $R$ by $P(\mu \rightarrow \mu)=\left(X^{2} R-1\right) /\left(X^{2} R-1+2 X-2 X R\right)$, where $X$ is the initial flux ratio $\nu_{\mu} / \nu_{e}$, as a function of neutrino energy and zenith angle. In Table 1 , for the atmospheric neutrinos, in addition to the raw double ratio $R$, we give the corrected value for the survival probability obtained from the above formula, together with our calculated values of the flux ratio used to make the correction.

Also in Table 1 , for the solar neutrinos, we give the KAMIOKA ( $\nu e \rightarrow \nu e)$ data point corrected for cross-talk effects in the presence of neutrino oscillations. The crosssection for $\nu_{\mu}$ (or $\nu_{\tau}$ ) scattering on electrons, through the neutral current interaction, is about a factor of six smaller than the cross-section for $\nu_{e}$ scattering on electrons, which proceeds via both charged and neutral current interactions. The corrected survival probability is then related to the measured raw ratio $S$ by $P(e \rightarrow e)=(6 S-1) / 5$, where in this case the correction formula is independent of the assumed form of the mixing. No cross-talk corrections are required in the case of the chlorine or gallium experiments which measure $P(e \rightarrow e)$ directly.

The corrected values for the atmospheric and solar data are displayed in Figure 2, 
together with the accelerator and reactor data as before. The data are now in very satisfactory agreement with the expected (solid) curve, with the sole exception of the HOMESTAKE solar neutrino point, which shows a deviation of about four standard deviations. The (corrected) solar data are shown on an expanded scale in Figure 3. Without the data from the gallium experiments, the HOMESTAKE point, which is clearly consistent with $P(e \rightarrow e)=1 / 3$, could easily have been fitted by appropriately positioning the second threshold. In Figure 3 this is illustrated by the broken curve which corresponds to $\Delta m^{\prime 2} \simeq 1.6 \times 10^{-11} \mathrm{eV}^{2}$ and which has been calculated assuming $\pm 25 \%$ averaging, so as to simulate the energy spread in a solar experiment. It should be pointed out, however, that with regard to the solar neutrino data, the two gallium results are by far the most reliable, firstly because the experimental efficiency has been measured directly using a laboratory neutrino source, and secondly because the calculated rates for p-p neutrinos are only weakly dependent on solar model parameters. We have also considered the effect of matter oscillations in the Sun (the MSW [24] effect) [25]. We have verified that (as one might perhaps expect), for $\Delta m^{2} \sim 10^{-2} \mathrm{eV}^{2}$ and in the specific case of maximal mixing, matter oscillations in the Sun do nothing to modify the vacuum predictions for the $\nu_{e}$ survival probability, for any value of $\Delta m^{\prime 2}$. In particular, they do not help to resolve the discrepancy between the HOMESTAKE result and the bulk of the data, within the present context.

A fit to the data of Figure 2, excluding the HOMESTAKE point, yields $\Delta m^{2}=$ $(0.72 \pm 0.18) \times 10^{-2} \mathrm{eV}^{2}$ with $\chi^{2} / \mathrm{DOF}=19.2 / 26$. (Including the HOMESTAKE point yields $\chi^{2} / \mathrm{DOF}=36.1 / 27$, and the same fitted value of $\Delta m^{2}$.) A fit for $\Delta m^{\prime 2}$, excluding the HOMESTAKE point, yields $\Delta m^{\prime 2}<0.9 \times 10^{-11} \mathrm{eV}^{2}$ at $90 \%$ confidence (and no limit if the HOMESTAKE point is included). Whilst these results are readily interpreted in terms of a hierarchical spectrum for the neutrino masses, we emphasise that the data only require a hierarchy for the mass-squared differences, and do not, for example, exclude a mass spectrum with a substantial mass-squared offset [26]. Neither do they exclude the possibility that the usual hierarchy of mass-squared differences is inverted here, ie. $\Delta m_{12}^{2} \simeq-\Delta m_{31}^{2}\left(\equiv \Delta m^{2}\right)$ and $\Delta m_{23}^{2}\left(\equiv \Delta m^{\prime 2}\right) \ll \Delta m^{2}$. On the (minimal) assumption that the neutrino mass spectrum is qualitatively similar to that of the charged leptons and quarks, our results can be re-expressed in terms of the neutrino masses as follows: $m_{3} \simeq 85 \pm 10 \mathrm{meV}$ and $m_{1}, m_{2}<3 \mu \mathrm{eV}$ at $90 \%$ confidence. With the first and second generation neutrinos being so nearly degenerate ( $\Delta m^{\prime 2} \equiv 0$ is not excluded by the present data), it is doubtful whether oscillation effects involving them (eg. CP violating asymmetries) will ever be observed, though conceivably neutrino pulses from supernovae could be used.

The above results are consistent with existing upper limits on other lepton number violating processes such as $\mu \rightarrow e \gamma$. Assuming maximal mixing and $\Delta m^{2}=0.72 \times 10^{-2}$ 
$\mathrm{eV}^{2}$, we calculate the branching ratio: $B(\mu \rightarrow e \gamma) \simeq 3 \times 10^{-53}$ [27]. The current experimental upper limit is: $B(\mu \rightarrow e \gamma)<4.9 \times 10^{-11}$ [28]. Similarly neutrino decay, for example $\nu_{3} \rightarrow \nu_{2} \gamma$, has a mean lifetime of $4 \times 10^{42}$ yrs. Lepton number violating decays are therefore presumably unobservable.

If the above hypothesis is correct, a number of definite predictions should be verified in future experiments. Longer baseline reactor experiments, such as SAN ONOFRE [29] and CHOOZ [30], would seem to be a logical next step. In Figure 4 we show the ILL/GOSGEN and BUGEY data in fine bins of $L / E$ together with the full oscillation curve (ie. with no averaging) corresponding to $\Delta m^{2}=0.72 \times 10^{-2} \mathrm{eV}^{2}$ and maximal mixing. With well defined $L$ and $E$ one can realistically expect to map out the details of the full oscillation curve. Note that a pronounced minimum in the counting rate, corresponding to $P(\bar{e} \rightarrow \bar{e})=1 / 9$, is expected for $L / E \simeq 170 \mathrm{~m} / \mathrm{MeV}$. Proposed long baseline accelerator experiments, FNAL/SOUDAN [31], CERN/GRAN-SASSO [32] and KEK/SUPER-KAMIOKA [33], with $L / E \simeq 50-100 \mathrm{~km} / \mathrm{GeV}$, will be equally important. In particular, it should be possible to verify that the rate of $\nu_{e}$ events accounts for only one-half of the loss of $\nu_{\mu}$. events, and for $E \gtrsim 10 \mathrm{GeV}$ the prediction $P(\mu \rightarrow e)=P(\mu \rightarrow \tau)$ can be verified directly, if the detectors are able to identify $\tau$ leptons. On the other hand, short baseline, high flux accelerator experiments $(L / E \simeq$ $0.05 \mathrm{~km} / \mathrm{GeV}$ ) such as CHORUS [34] and NOMAD [35] will be sensitive to small mixing angles but require large masses, and would be expected to yield a null result $\left(P(\mu \rightarrow \tau)<10^{-6}\right)$. In a few years therefore, a range of experiments should be able to confirm or refute the hypothesis of threefold maximal mixing for leptons.

\section{Acknowledgement}

It is a pleasure to thank R. Bingham and P. J. Litchfield for helpful comments.

\section{References}

[1] R. Davis et al., Proc. 21st Int. Cosmic Ray Conf. Univ. of Adelaide. Vol. 12 (1990) 143. (ed. R. J. Protheroe).

[2] P. Anselmann et al. Phys. Lett. B285 (1992) 376. Phys. Lett. B285 (1992) 390. Phys. Lett. B314 (1993) 445.

[3] A. I. Abazov at al. Phys. Rev. Lett. 67 (1991) 3332.

[4] K. S. Hirata et al. Phys. Rev. Lett. 65 (1990) 1297.

Phys. Rev. Lett. 66 (1991) 9. Phys. Rev. D44 (1991) 2241.

[5] K. S. Hirata et al. Phys. Lett. B205 (1988) 416. Phys. Lett. B280 (1992) 146.

Y. Fukada et al. Phys. Lett. B335 (1994) 237. 
[6] P. J. Litchfield. Proc. Int. Europhys. Conf. on High Energy Physics. Marseille (1993) 557. (Editions Frontieres, ed. J. Carr and M. Perrottet.)

[7] R. Becker-Szendy et al. Phys. Rev. D46, (1992) 3720.

D. Casper et al. Phys. Rev. Lett. 66, (1992) 2561.

[8] P. F. Harrison and W. G. Scott. Phys. Lett. B333 (1994) 471.

[9] C. Jarlskog. Phys. Rev. Lett. 55 (1985) 1039.

[10] N. Cabibbo. Phys. Lett. B72 (1978) 333.

V. Barger, K. Whisnant and R. J. N. Phillips. Phys. Rev D24 (1981) 538.

J. N. Bahcall, Neutrino Astrophysics, Cambridge University Press (1989).

C. W. Kim and J. A. Lee. JHU-TIPAC-930023 (1993) (unpublished).

[11] P. Berge et al. Z. Phys. C35 (1987) 443.

[12] A. J. Buras, Rev. Mod. Phys. Vol 52 (1980) 199.

N. J. Baker et al. Phys. Rev. D25 (1982) 617.

[13] F. Bergsma et al. Phys. Lett. B142 (1984) 103.

[14] B. Bodman et al. Phys. Lett. B280 (1992) 198.

[15] J.-L. Vuilleumier et al. Phys. Lett. B114 (1982) 298.

K. Gabathuler et al. Phys. Lett. B138 (1984) 449.

V. Zacek et al. Phys. Lett. B164 (1985) 193.

G. Zacek et al. Phys. Rev. D34 (1986) 2621.

[16] Y. Declais. Review Talk at 6th International Workshop on Neutrino Telescopes. Venice (1994). LAPP-EXP-94.08.

[17] G. S. Vidyakin et al. JETP Lett. 55 (1992) 206; JETP Lett. 59 (1994) 391.

[18] G. Barr, T. K. Gaisser and T. Stanev, Phys. Rev. D39 (1989) 3532.

H. Lee and Y. S. Koh, Nuov. Cim. 105 B (1990) 883.

M. Honda et al. Phys. Lett. B 248 (1990) 193.

D. H. Perkins. Astroparticle Physics. 2 (1994) 1.

[19] C. Berger et al. Phys. Lett. B 227 (1989) 489. B 245 (1990) 305.

[20] M. Aglietta, et al. Europhys Lett. 15, (1991) 559.

[21] D. H. Perkins, Nucl. Phys. B399 (1993) 3. 
[22] E. Bellotti, Proc. 27th Int. Conf. High Energy Phys. Glasgow (1994). Inst. of Phys. London.

[23] J. N. Bahcall and M. H. Pinsonneault, Rev. Mod. Phys. 64 (1992) 885.

S. Turck-Chieze et al., Phys. Rep. 230 (1993) 57.

[24] L. Wolfenstein Phys. Rev. D17 (1978) 2369. Phys. Rev. D20 (1979) 2634.

S. P. Mikheyev and A. Yu. Smirnov. Il Nuovo Cimento 9C (1986) 17.

[25] V. Barger et al. Phys. Rev. D22 (1980) 2718.

H. A. Bethe Phys. Rev. Lett. 56 (1986) 1305.

S. P. Rosen and J. M. Gelb, Phys. Rev. D34 (1986) 969, D39 (1989) 3190.

L. M. Krauss, E. Gates and M. White. Phys. Lett. 299B (1993) 94.

[26] D. O. Caldwell and R. N. Mohapatra, Phys. Rev. D 46 (1993) 3259. UCSB-HEP-94-03 (1994).

[27] F. Boehm and P. Vogel. Physics of Massive Neutrinos.

Cambridge University Press (1987) 88.

[28] Particle Data Group. Particle Physics Booklet. American Inst. of Phys. (1994).

[29] R. G. H. Robertson, Proc. 26th Int. Conf. High Energy Physics. Dallas. Vol 1 (1992) 140. (American Inst. Phys. ed. J. R. Sandford)

F. Boehm. Particles, Strings and Cosmology. (1992) 96.

(World Scientific, ed. P. Nath and S. Reucroft.)

[30] Y. Declais et al. Search for Neutrino Oscillations at a Distance of $1 \mathrm{~km}$ from Two Power Reactors at Chooz. Letter of Intent. (1992).

[31] W. W. M. Allison et al. Fermilab Proposal P-822 (1993).

[32] C. Rubbia CERN-PPE/93-08.

[33] K. Nishikawa INS-Rep-924 (1992).

[34] J. Brunner, Proc. Int. Europhys. Conf. on High Energy Physics. Marseille (1993) 555. (Editions Frontieres, ed. J. Carr and M. Perrottet.)

[35] CERN Annual Report, Vol. 2 (1993) 14. 


\begin{tabular}{|c|c|c|c|c|c|}
\hline Experiment & $\nu_{\mu} / \nu_{e}$ & Energy & Distance & Measured & Corrected \\
\hline $\begin{array}{l}\text { CDHS-SPS [11] } \\
\text { CHARM-PS [13] } \\
\text { KARMEN [14] }\end{array}$ & $\begin{array}{l}\nu_{\mu} \\
" \\
" \\
\nu_{e}\end{array}$ & $\begin{array}{l}E=120 \mathrm{GeV} \\
E=50 \mathrm{GeV} \\
E \simeq 1.3 \mathrm{GeV} \\
E \simeq 40 \mathrm{MeV}\end{array}$ & $\begin{aligned} L & =0.6 \mathrm{~km} \\
& \geqslant \\
L & =0.8 \mathrm{~km} \\
L & =17.7 \mathrm{~m}\end{aligned}$ & $\begin{array}{l}1.00 \pm 0.04 \\
0.98 \pm 0.03 \\
0.96 \pm 0.08 \\
1.05 \pm 0.12\end{array}$ & $\begin{array}{l}- \\
- \\
- \\
-\end{array}$ \\
\hline $\begin{array}{l}\text { ILL/GOSGEN [15] } \\
\text { BUGEY [16] } \\
\text { KRASNOYARSK } \\
\text { [17] }\end{array}$ & $\begin{array}{l}\bar{\nu}_{e} \\
" \\
" \\
" \\
" \\
" \\
" \\
" \\
"\end{array}$ & $\begin{array}{c}E \simeq 5 \mathrm{MeV} \\
" \\
" \\
" \\
" \\
" \\
" \\
" \\
"\end{array}$ & $\begin{aligned} L & =8.8 \mathrm{~m} \\
L & =37.9 \mathrm{~m} \\
L & =45.9 \mathrm{~m} \\
L & =64.7 \mathrm{~m} \\
L & =15 \mathrm{~m} \\
L & =40 \mathrm{~m} \\
L & =95 \mathrm{~m} \\
L & =57 \mathrm{~m} \\
L & =231 \mathrm{~m}\end{aligned}$ & $\begin{array}{l}0.96 \pm 0.12 \\
1.02 \pm 0.02 \\
1.05 \pm 0.02 \\
0.98 \pm 0.05 \\
0.98 \pm 0.05 \\
1.00 \pm 0.05 \\
0.90 \pm 0.13 \\
0.99 \pm 0.05 \\
1.16 \pm 0.21\end{array}$ & $\begin{array}{l}- \\
- \\
- \\
- \\
- \\
- \\
- \\
-\end{array}$ \\
\hline $\begin{array}{l}\text { KAMIOKA [5] } \\
\text { (sub-GeV) } \\
\text { IMB [7] } \\
\text { FREJUS [19] } \\
\text { NUSEX [20] } \\
\text { SOUDAN [6] }\end{array}$ & $\begin{array}{c}4.5 / 1 \\
3.2 / 1 \\
2.2 / 1 \\
3.2 / 1 \\
4.5 / 1 \\
2.1 / 1 \\
" \\
" \\
" \\
" \\
"\end{array}$ & $\begin{array}{c}E \simeq 5 \mathrm{GeV} \\
" \\
" \\
" \\
" \\
E \simeq 0.5 \mathrm{GeV} \\
" \\
" \\
E \simeq 1 \mathrm{GeV} \\
" \Rightarrow \\
E \simeq 0.5 \mathrm{GeV}\end{array}$ & $\begin{aligned} & L \simeq 25 \mathrm{~km} \\
& L \simeq 50 \mathrm{~km} \\
& L \simeq 500 \mathrm{~km} \\
& L \simeq 5000 \mathrm{~km} \\
& L \simeq 10000 \mathrm{~km} \\
& L \simeq 40 \mathrm{~km} \\
& L \simeq 6400 \mathrm{~km} \\
& L \simeq 500 \mathrm{~km} \\
& " ~ \\
& " \\
& "\end{aligned}$ & $\begin{array}{l}1.27^{+0.61} \\
0.63^{+0.21}-0.38 \\
0.51^{+0.15}+.16 \\
0.46^{+0.18} \\
0.28^{+0.10 .12} \\
0.59 \pm 0.07 \\
0.62 \pm 0.10 \\
0.54 \pm 0.13 \\
0.87 \pm 0.18 \\
0.99 \pm 0.32 \\
0.69 \pm 0.21\end{array}$ & $\begin{array}{l}1.11^{+0.16} \\
0.70^{+0.18}-16 \\
0.41^{+0.18} \\
0.52^{+0.19}-17 \\
0.42^{+0.13} .0 .15 \\
0.48 \pm 0.13 \\
0.52 \pm 0.13 \\
0.42 \pm 0.17 \\
0.84 \pm 0.23 \\
0.99 \pm 0.40 \\
0.61 \pm 0.27\end{array}$ \\
\hline $\begin{array}{l}\text { KAMIOKA [4] } \\
\text { HOMESTAKE [1] } \\
\text { SAGE [3] } \\
\text { GALLEX [2] }\end{array}$ & $\begin{array}{l}\nu_{e} \\
" \\
" \\
"\end{array}$ & $\begin{array}{l}E \simeq 10 \mathrm{MeV} \\
E \simeq 5 \mathrm{MeV} \\
E \simeq 0.5 \mathrm{MeV} \\
\quad "\end{array}$ & $\begin{aligned} L= & 1.5 \times 10^{11} \mathrm{~m} \\
& " \\
& " \\
& "\end{aligned}$ & $\begin{array}{l}0.58 \pm 0.11 \\
0.35 \pm 0.05 \\
0.54 \pm 0.10 \\
0.62 \pm 0.09\end{array}$ & $\begin{aligned} & 0.50 \pm 0.13 \\
&- \\
&- \\
&-\end{aligned}$ \\
\hline
\end{tabular}

Table 1: Results for the survival probability $P(l \rightarrow l)$ measured in disappearance experiments at accelerators and reactors, together with the results from atmospheric and solar neutrino experiments. In addition to the raw measurement, the corrected value of $P(l \rightarrow l)$ is given where appropriate (see text for details of corrections). The errors quoted are the total errors from all known sources, statistical and systematic. The beam composition, energy and propagation length for each experiment are also given. 


\section{Figure Captions}

Figure 1: Results for the survival probability $P(l \rightarrow l)$ measured in disappearance experiments at accelerators and reactors, together with the raw measured results (see text) from atmospheric and solar neutrino experiments, plotted as a function of $L / E$. The data suggest a distinct constant plateau $(L / E>80 \mathrm{~km} / \mathrm{GeV})$ consistent with the prediction from maximal mixing, $P(l \rightarrow l)=5 / 9$. Maximal mixing predicts a universal $L / E$ dependence as shown by the solid curve, which has been calculated for $\Delta m^{2}=0.72 \times 10^{-2} \mathrm{eV}^{2}$ and $\Delta m^{\prime 2}=0$ (with $\pm 100 \%$ averaging, see text). The upper horizontal scale gives $\sqrt{\Delta m^{2}}$ (at the point where the plateau value is first crossed).

Figure 2: Results for the survival probability $P(l \rightarrow l)$ measured in disappearance experiments at accelerators and reactors, together with the corrected results (see text) from atmospheric and solar neutrino experiments, plotted as a function of $L / E$. With the exception of the HOMESTAKE solar neutrino point, all the data are consistent with threefold maximal mixing and a fit (with $\left.\Delta m^{\prime 2} \equiv 0\right)$ to all the data (excluding the HOMESTAKE point) yields $\Delta m^{2}=(0.72 \pm 0.18) \times 10^{-2}$ $\mathrm{eV}^{2}$, as shown by the solid curve.

Figure 3: The solar data in more detail. The solid curve shows the maximal mixing prediction $P(l \rightarrow l)=5 / 9$. Without the two gallium points, the HOMESTAKE point (which is consistent with $P(l \rightarrow l)=1 / 3)$ could have been fitted by positioning the second threshold appropriately, as illustrated by the broken curve which corresponds to $\Delta m^{\prime 2}=1.6 \times 10^{-11} \mathrm{eV}^{2}$ (with $\pm 25 \%$ averaging, see text). Excluding the HOMESTAKE point, the fit yields $\Delta m^{\prime 2}<0.9 \times 10^{-11} \mathrm{eV}^{2}$ at $90 \%$ confidence.

Figure 4: The first threshold region in more detail, showing the ILL/GOSGEN and BUGEY data in fine bins of $L / E$ together with the KRASNOYARSK data and the corrected atmospheric neutrino data. The solid curve is the predicted universal survival probability based on the fitted value $\Delta m^{2}=0.72 \times 10^{-2} \mathrm{eV}^{2}$ (with $\pm 100 \%$ averaging, see text). The broken curve is calculated with no averaging and shows a pronounced dip at $L / E \simeq 170 \mathrm{~m} / \mathrm{MeV}$, which should be observable in long baseline experiments at reactors and accelerators. 


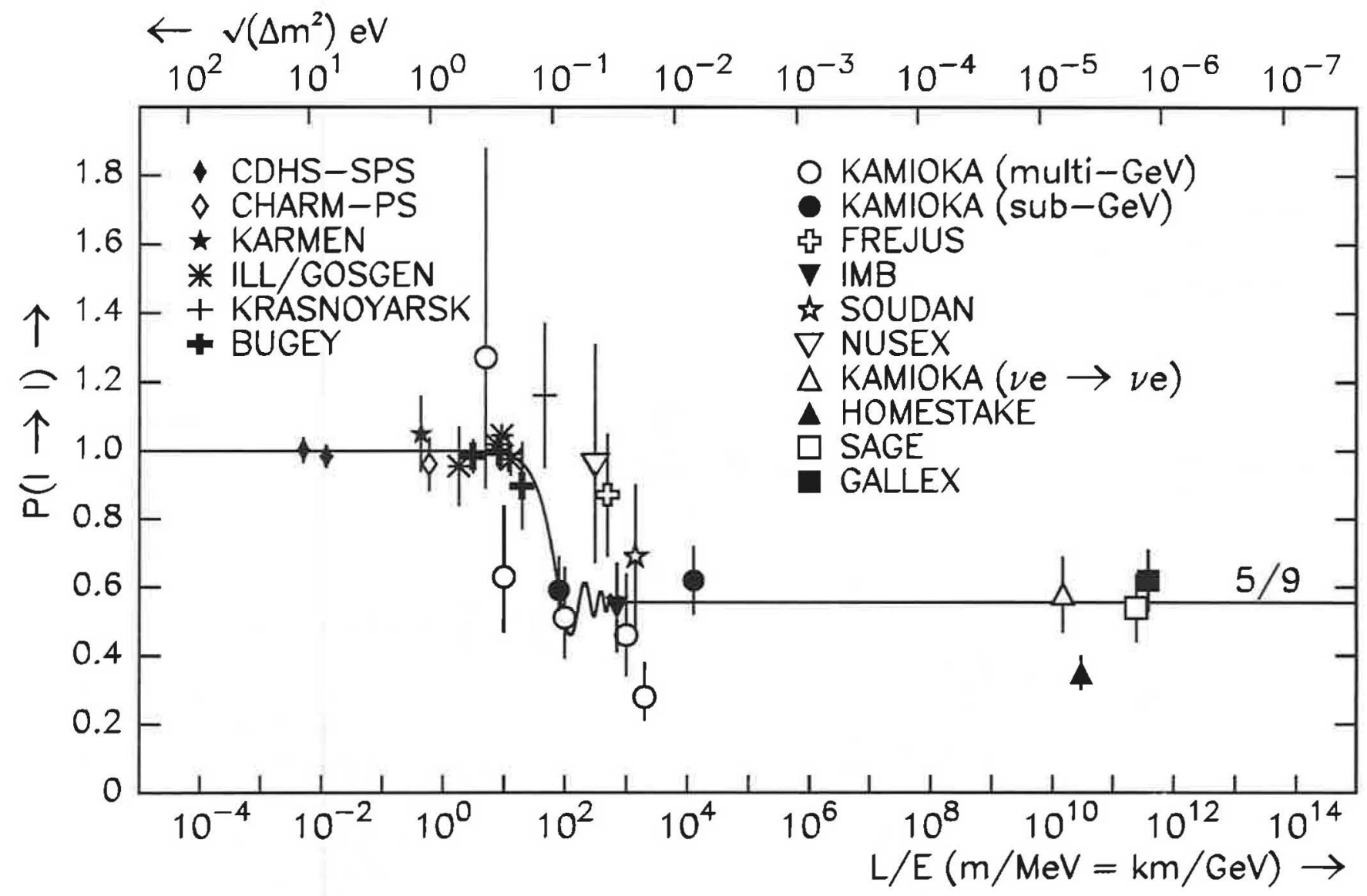

Figure 1 


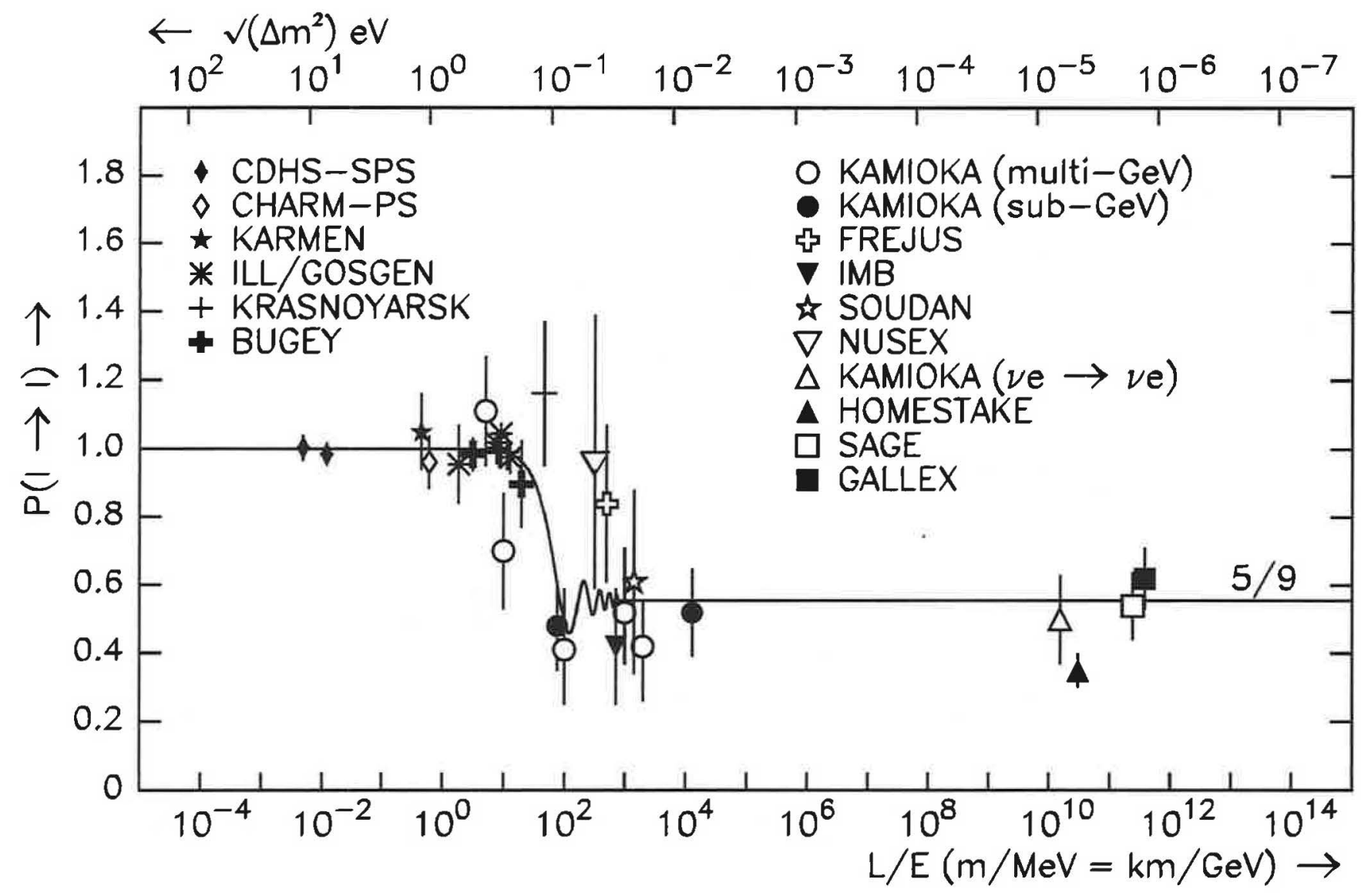

Figure 2 


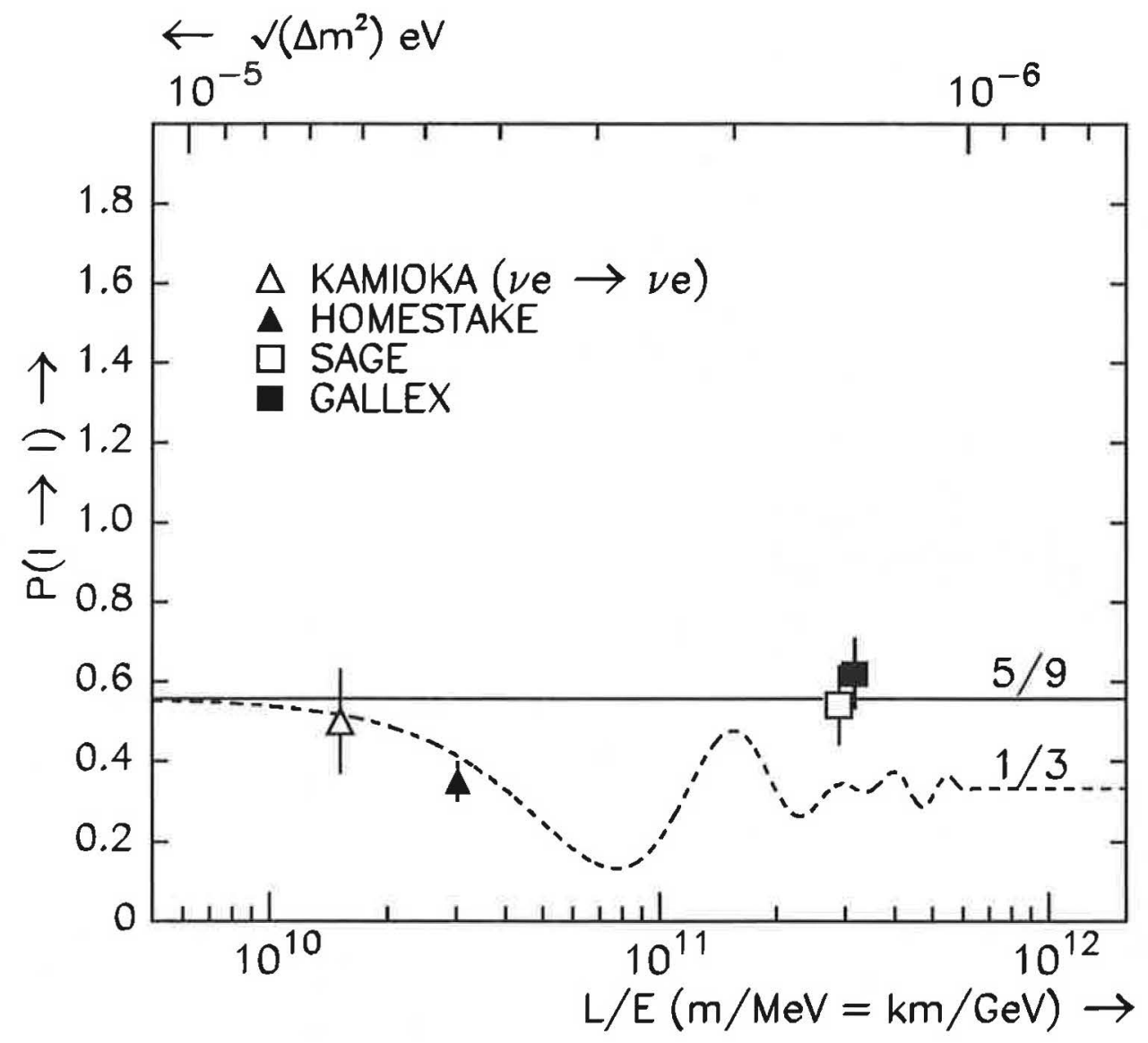

Figure 3 


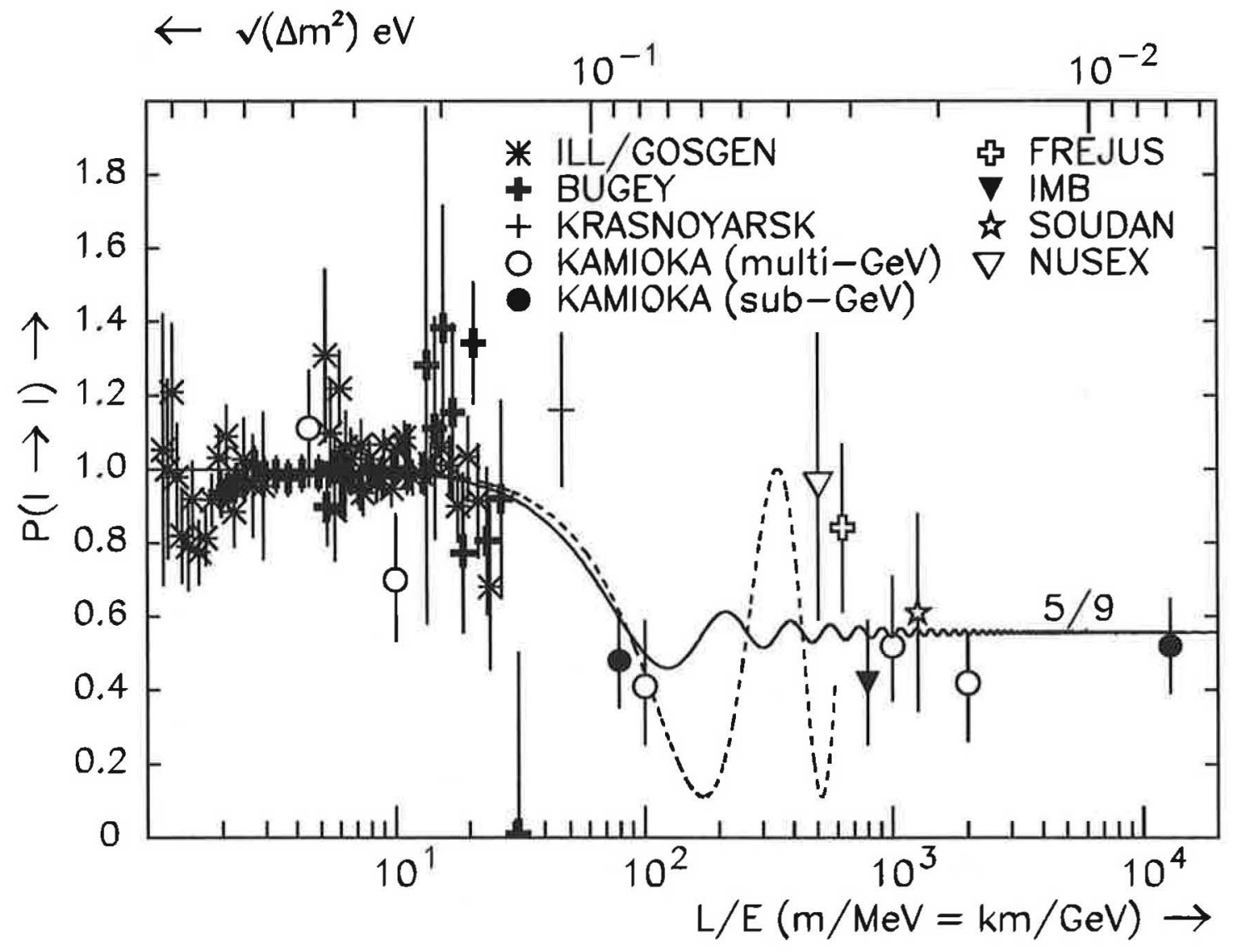

Figure 4 
